\title{
ALKYLATION OF METHYL 3-HYDROXYINDOLE-2-CARBOXYLATE USE IN PYRIMIDINE SYNTHESIS
}

\section{A.S. Bourlot, J.Y. Merour *.}

LCBA, URA-CNRS 499, Universite d'Orleans, BP 6759, 45067 Orleans Cedex 2, France.

\begin{abstract}
Three alkylation sites (O,C-2 or $\mathrm{N}$ atoms) are present on methyl 3-hydroxyindole-2-carboxylate $4 \mathrm{a}$. Functionalized halides were used to study the regioselectivity of these alkylations. In order to illustrate this work, the pyrimidine 17 has been synthesized from the $O$-alkylated compound $13 a$.
\end{abstract}

Introduction : 3-Hydroxyindole-2-carboxylic acid derivatives 1 represent well-known and readily available compounds (1). Compounds 1 are used as synthons for the preparation of indole-fused heterocycles, incorporating the 3hydroxy group in ring formation to give compounds of potential medicinal interest, e.g. 2 (anti-allergy and/or CNS activities) (2,3). Recently, Hirota et al. reported that some 4-alkylamino-6,7-dihydro-5H-pyrimido[5,4-d][1]benzazepines 3a or 5,6-dihydro[1]benzoxepino[5,4-ofpyrimidin-4(3H)-one $3 \mathrm{~b}(6)$ show stronger inhibitory activity against collagen-induced aggregation of rabbit blood platelets than aspirin, which is an anti-platelet agent $(6,7)$. Our goal was to replace the benzo part of compound $\mathbf{3 b}$ by an indole moiety.<smiles>[R]c1cc2c(O)c(C(=O)O)n(P)c2cc1Br</smiles><smiles>CCn1c2ccccc2c2oc(C(=O)O)cc(=O)c21</smiles>

2<smiles>Nc1ncnc2c1CCNCc1ccccc1-2</smiles>

$3 a$<smiles>O=c1[nH]cnc2c1CCOc1ccccc1-2</smiles>

3b

Methyl 3-hydroxyindole-2-carboxylate may exist in two tautomeric forms, $4 a$ and $\mathbf{4 b}$ 
<smiles>CC(=O)c1[nH]c2ccccc2c1O</smiles>

$4 a$<smiles>CC(=O)C1Nc2ccccc2C1=O</smiles>

4b

The reactivity of the ketoester is mainly governed by the enolic form $4 \mathbf{4 a} ; \mathbf{4 a}$ is soluble in aqueous sodium hydroxide and precipitated with acid. In $\mathrm{CDCl}_{3}$ solution, $\mathbf{4 a}$ is the only present tautomeric form. Such a behaviour has also been reported for the corresponding 3-hydroxy-1H-indol-2-yl ethanone (4).

Three alkylation sites are present in compound $4 \mathrm{a}$, so $\mathrm{O}, \mathrm{C}$ or $\mathrm{N}$-alkylation can occur. Polar aprotic solvents and large cations favour $O$-alkylation; according to the HSAB theory, iodo compounds give mainly $C$-alkylation while sulfonates give $\mathrm{O}$-alkylation (8). For compound $\mathbf{4 a}$, the possibility of $\mathrm{N}$-alkylation also exists.

We have recently revisited the alkylation of methyl indole-2-carboxylate with the functionalized halides 5 and $\mathbf{8}$. In all cases the $N$-alkylation was observed (9).

Compound $4 \mathrm{a}$ has been previously $C$ and/or $O$-alkylated using allylic bromides $(10,11)$ and exclusively $O$ alkylated with methyl or allylic tosylates (10), with compound 5 b (15) or with 2-bromopropane (12). These 0 -alkylated compounds have been $N$-phenylated (Ullmann-type reaction) (12). The methoxycarbonyl group of compound 4 a has been transformed into a $\beta$-keto sulfone group (4). Ethyl 3-hydroxy-1-methylindole-2-carboxylate 22 was not obtained by $N$ methylation of ethyl 3-hydroxyindole-2-carboxylate, but only by reacting $\mathrm{N}$-methylaniline with diethyl bromomalonate (5).

The present investigation was undertaken to examine the regioselectivity of the alkylation of $4 a$ with functionalized halides like $5,8,12$ in order to obtain diesters or cyanoesters, which could be submitted to a Dieckmann condensation; the products obtained are potential anti-platelet agents $(13,14)$. (The experimental conditions were $\mathrm{K}_{2} \mathrm{CO}_{3}$ as base, with 1 equivalent of halide, in DMF at $90^{\circ} \mathrm{C}$ ).

\section{Results :}<smiles>CC(=O)c1[nH]c2ccccc2c1O</smiles>

$4 a$<smiles>[R]OCc1c(C(=O)OC)n([TlH])c2ccccc12</smiles>

6a $\mathrm{R}=\mathrm{CH}_{2}$<smiles>[R]OCCOc1c(C(=O)OC)n(C)c2ccccc12</smiles>

7a $\mathrm{R}=\mathrm{CH}_{3}$ 7b $\mathrm{R}=\mathrm{C}_{2} \mathrm{H}_{5}$

Compound $\mathbf{4 a}$, treated with 1 equivalent of methyl chloroacetate $\mathbf{5 a}$, led to a mixture of 0 -alkylated compound $6 \mathbf{a}$. and $\mathrm{N}, \mathrm{O}$-disubstituted compound $7 \mathrm{a}$. The treatment of $\mathbf{4 a}$ with 1 equivalent of ethyl bromoacetate $5 \mathrm{~b}$ gave only the $\mathrm{N}, \mathrm{O}$ disubstituted product $7 \mathbf{b}$. The same disubstituted compound $7 \mathbf{b}$ was obtained if only 0.5 equivalent of $5 \mathbf{b}$ was used. 
The reaction of $\mathbf{4 a}$ with the halides 5 allowed us to obtain only the $\mathrm{O}$ - and/or $\mathrm{N}, \mathrm{O}$-dialkylated compounds; meanwhile the yields are low in isolated products after purification on silica gel chromatography $(<20 \%)$. It has been reported that much higher yields result using acetone instead of DMF as solvent, in the O-alkylation of ethyl 3hydroxyindole-2-carboxylate with $5 b$ (15). 1-Chloro-2-propanone in refluxing acetone led also in good yield (74\%) to the 0 alkylated compound (16). Hirota et al. also reported the O-alkylation of 3-hydroxybenzo[b]thiophene-2-carbonitrile, using $\mathrm{K}_{2} \mathrm{CO}_{3}$ and chloroacetonitrile, in acetone in $70 \%$ yield (17).

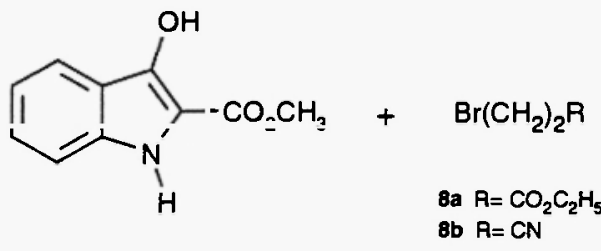

$4 a$<smiles>CC(=O)C1(C)Nc2ccccc2C1=O</smiles>

9a $\mathrm{R}=\mathrm{CO}_{2} \mathrm{C}_{2} \mathrm{H}_{5}$ 9b $\mathrm{R}=\mathrm{CN}$

The incorporation of one more methylene unit in the halides $\mathbf{8}$ resulted in a selective $C$-alkylation of compound 4a with much higher yield : indeed, reaction of compound $4 a$ with ethyl 3-bromopropanoate 8 a gave the product $9 a$ in $65 \%$ yield, and reaction with 3 -bromopropanenitrile $8 \mathrm{~b}$ led to the product $9 \mathrm{~b}$ in $67 \%$ yield.<smiles>O=C(O)c1[nH]c2ccccc2c1O</smiles>

$4 a$

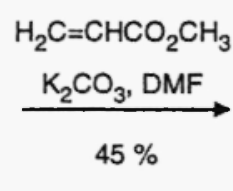

$45 \%$<smiles>COC(C)=O</smiles>

10<smiles>CC(=O)C12CCC(=O)N1c1ccccc1C2=O</smiles>

11

The Michael addition of compound $4 \mathrm{a}$ on methyl acrylate led to a mixture of the C-alkylated product 10 in $20 \%$ yield, and of the tricyclic compound 11 in $25 \%$ yield. Compound 11 was the result of the intramolecular acylation of 10 . Its structure has been elucidated using 2D NMR and by an independent synthesis (16)).

We switched from C-alkylation to O-alkylation by using the halides 12 with one additional methylene unit.

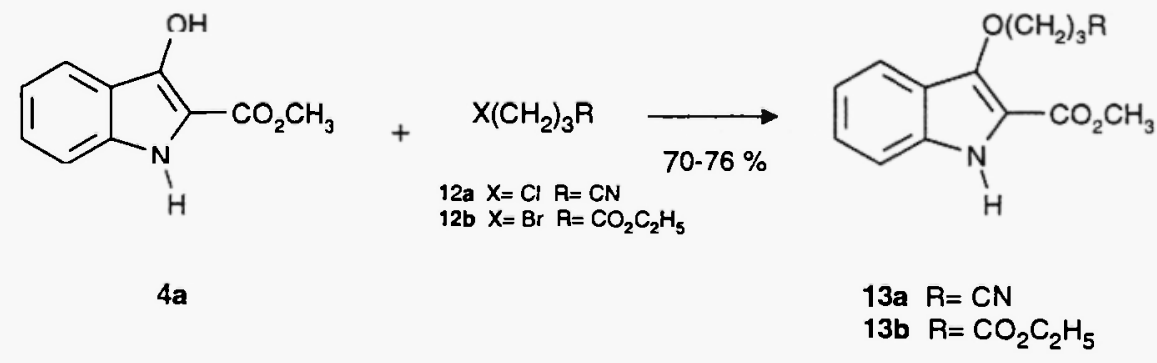

Action of 4-chlorobutanenitrile 12a with compound 4a gave the O-alkylated product 13a in 76\% yield, and action of ethyl 4-bromobutanoate $12 \mathrm{~b}$ led similarly to the 0 -alkylated compound $13 \mathrm{~b}$ in $70 \%$ yield. We have observed in both 
cases a trace of the $\mathrm{N} ; \mathrm{O}$-disubstituted compounds 14 . The use of refluxing acetone, instead of DMF, gave much lower yields of $O$-alkylated products $13 a, b$.

Using 5 eq. of 4 -chlorobutanenitrile 12a with compound $4 \mathbf{a}$, in refluxing acetonitrile ( $48 \mathrm{~h}$ ), led to the $\mathrm{N}, \mathrm{O}$ dialkylated product $14 \mathrm{a}$ in $50 \%$ yield. Compound 14 a was also obtained by $\mathrm{N}$-alkylation of $13 \mathrm{a}$ with 4 -chlorobutanenitrile. Compound $14 \mathrm{~b}$ was similarly obtained from $4 \mathrm{a}$ using an excess of $12 \mathrm{~b}$ ( 4 eq.), in $60 \%$ yield (18).<smiles>COC(=O)c1c(OC)c2ccccc2n1CC=P</smiles>

14a $\mathrm{R}=\mathrm{CN}$

14b $\mathrm{R}=\mathrm{CO}_{2} \mathrm{C}_{2} \mathrm{H}_{5}$<smiles>COC(=O)c1c(OC)c2ccccc2n1CC#N</smiles>

24

In connection with molecules which might have potential biological activities $(6,7,19)$, we describe here the use of compound 13a in the synthesis of pyrimidine derivative 17 (Scheme 1):<smiles>CCOc1c(C(=O)OC)[nH]c2ccccc12</smiles>

13a $\mathrm{R}=\mathrm{CN}$

13b $\mathrm{R}=\mathrm{CO}_{2} \mathrm{C}_{2} \mathrm{H}_{5}$<smiles>[R]C1CCOc2c(n(C)c3ccccc23)C1=O</smiles>

16a $\mathrm{R}=\mathrm{CN}$

16b $\mathrm{R}=\mathrm{CO}_{2} \mathrm{C}_{2} \mathrm{H}_{5}$

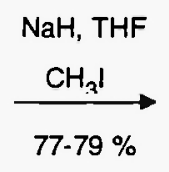<smiles>CCOc1c(C(=O)OCc2ccccc2)n(C)c2ccccc12</smiles>

15a $R=C N$

15b $\mathrm{R}=\mathrm{CO}_{2} \mathrm{C}_{2} \mathrm{H}_{5}$

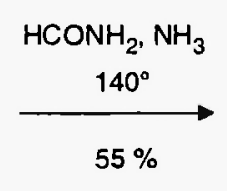<smiles>[R7]Nc1ncnc2c1CCOc1c-2n(C)c2ccccc12</smiles>

$17 \mathrm{R}=\mathrm{H}$

$20 \mathrm{R}=\mathrm{COCH}_{2}$

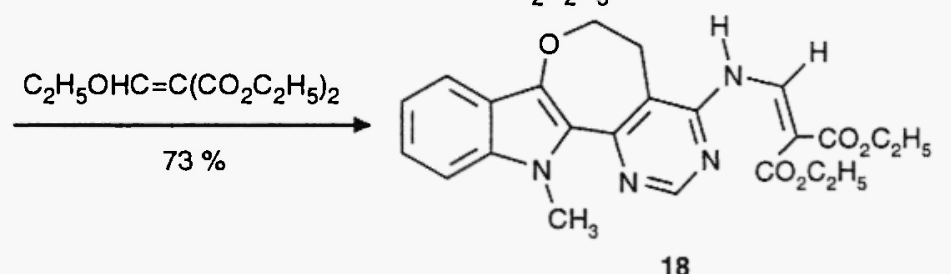

$\stackrel{18}{\longrightarrow}$ $86 \%$<smiles>CCOC(=O)c1cnc2c3c(ncn2c1=O)-c1c(c2ccccc2n1C)OCC3</smiles>

19 
Methylation of compounds 13a,b with iodomethane gave the $\mathrm{N}$-methyl compounds $15 \mathrm{a}, \mathrm{b}$. Structure of compound 15a has been confirmed by treating ethyl 3-hydroxy-1-methylindole-2-carboxylate 22 with 4-chlorobutanenitrile 12a under the same conditions of alkylation as for $4 a$ : compound 23 was isolated in $76 \%$ yield and its NMR data were in agreement with those of compound 15a. Similarly, alkylation of methyl 3-methoxyindole-2-carboxylate with the halide 12a afforded the $\mathrm{N}$-alkylated compound $24\left(\delta_{\mathrm{NCH}_{2}}=4.55 \mathrm{ppm}\right)(16)$.<smiles>CCOC(=O)c1c(O)c2ccccc2n1C</smiles>

22

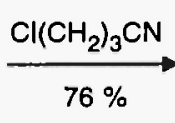<smiles>CCOC(=O)c1c(OC)c2ccccc2n1C</smiles>

23

After unsuccessful attempts ( $\mathrm{Na} / \mathrm{xylene}$, tBuOK, NaH/THF), the Dieckmann-type cyclization of compounds 15a,b was performed using LDATHF at low temperature to give $16 a, b$ in good yields. This cyclization can also be directly performed on compound $13 \mathrm{a}$ in $72 \%$ yield. The ketonitrile 16 a was either $O$-alkylated with methyl tosylate, or $C$-alkylated with methyl iodide. Reactivity of the ketoester $16 \mathrm{~b}$ ( $C$-alkylation, decarboxylation) will be presented later. The $\beta$-oxo nitrile $16 a$ was then cyclized by the reaction with formamide under ammonia stream at $140-150^{\circ} \mathrm{C}$ to give the compound 17 with a pyrimidine ring in $55 \%$ yield.

In order to study the reactivity of pyrimidine derivative 17, diethyl ethoxymethylenemalonate was reacted with compound 17 (14) to give the compound 18 in $73 \%$ yield, which was cyclized in diphenyl ether at $250^{\circ} \mathrm{C}$ to afford compound 19 , as shown in Scheme 1. The pyrimidine derivative 17 treated with acetyl chloride led to the compound 20 in $78 \%$ yield.

Conclusions: We have described a number of examples in which methyl 3-hydroxyindole-2-carboxylate 4a shows specific alkylation sites depending of the number of methylene units of the halide used. Further comprehensive studies of the alkylation of compound $\mathbf{4 a}$ are currently under investigation and will be reported in due course. Additionaly, these results allowed us to obtain the pharmacological interesting pyrimidine derivative 17.

\section{Experimental :}

Melting points were measured using a Kofler apparatus and are uncorrected. IR Spectra were recorded on a Perkin-Elmer 257 spectrometer, ${ }^{1} \mathrm{H}$ and ${ }^{13} \mathrm{C}$-NMR spectra were recorded on a Bruker AM 300 spectrometer using TMS as internal reference and mass spectra were recorded on a Nermag R-10-10C spectrometer. Column chromatography was carried out with Merck Kieselgel 60 ( 230-400 mesh ).

Alkylation of methyl 3-hydroxyindole-2-carboxylate; Typical procedure :

Methyl 3-methoxycarbonylmethoxyindole-2-carboxylate 6a : 
Ketoester $4 \mathrm{a}(0.250 \mathrm{~g}, 1.90 \mathrm{mmol})$ and $\mathrm{K}_{2} \mathrm{CO}_{3}(0.450 \mathrm{~g}, 5.13 \mathrm{mmol})$ were added to dry DMF (12 mL). Ethyl bromoacetate $(0.2 \mathrm{~mL}, 1.90 \mathrm{mmol})$ was then added dropwise, and the reaction mixture was stirred for $90 \mathrm{mn}$ at $90^{\circ} \mathrm{C}$. The solvent was evaporated under reduced pressure and the residue was quenched with $\mathrm{H}_{2} \mathrm{O}(15 \mathrm{~mL})$. The aq. layer was neutralized with a solution of $10 \% \mathrm{HCl}$ and extracted with $\mathrm{CH}_{2} \mathrm{Cl}_{2}(2 \times 10 \mathrm{~mL})$. The organic extracts were washed with $\mathrm{H}_{2} \mathrm{O}$, dried $\left(\mathrm{MgSO}_{4}\right)$ and evaporated under reduced pressure. The residue was chromatographed over silica gel using $\mathrm{CH}_{2} \mathrm{Cl}_{2}$ as eluent to give 6a; yield : $0.035 \mathrm{~g} \mathrm{(10 \% );} \mathrm{oil.} \mathrm{IR} \mathrm{(film)} \mathrm{v=3380} \mathrm{(br} \mathrm{NH),} \mathrm{1760,} 1680(\mathrm{C}=\mathrm{O}) \mathrm{cm}^{-1} .{ }^{1} \mathrm{H}-\mathrm{NMR}\left(\mathrm{CDCl}_{3}\right) \delta=3.71\left(\mathrm{~s}, 3 \mathrm{H}, \mathrm{OCH}_{3}\right)$, $3.89\left(\mathrm{~s}, 3 \mathrm{H}, \mathrm{OCH}_{3}\right), 4.87\left(\mathrm{~s}, 2 \mathrm{H}, \mathrm{OCH}_{2}\right), 7.05-7.36\left(\mathrm{~m}, 3 \mathrm{H}, \mathrm{H}_{\text {arom }}\right), 7.81(\mathrm{~d}, 1 \mathrm{H}, \mathrm{J}=7.8, \mathrm{H}-4)$.

Methyl 3-methoxycarbonylmethoxy-1-methoxycarbonylmethylindole-2-carboxylate $7 \mathbf{a}$ :

Yield : $0.030 \mathrm{~g}(7 \%)$; oil. IR (film) v=1760, $1680(\mathrm{C}=\mathrm{O}) \mathrm{cm}^{-1} .{ }^{1} \mathrm{H}-\mathrm{NMR}\left(\mathrm{CDCl}_{3}\right) \delta=3.69\left(\mathrm{~s}, 3 \mathrm{H}, \mathrm{OCH}_{3}\right), 3.77(\mathrm{~s}, 3 \mathrm{H}$, $\left.\mathrm{OCH}_{3}\right), 3.88\left(\mathrm{~s}, 3 \mathrm{H}, \mathrm{OCH}_{3}\right) 4.75\left(\mathrm{~s}, 2 \mathrm{H}, \mathrm{OCH}_{2}\right), 5.16\left(\mathrm{~s}, 2 \mathrm{H}, \mathrm{NCH}_{2}\right), 7.10(\mathrm{dd}, 1 \mathrm{H}, \mathrm{J}=7.5,7.9, \mathrm{H}-5), 7.19(\mathrm{~d}, 1 \mathrm{H}, \mathrm{J}=8.3, \mathrm{H}-$ 7), 7.30 (dd, $1 \mathrm{H}, J=7.5,8.2, H-6$ ), 7.80 (d, $1 \mathrm{H}, \mathrm{J}=7.9, \mathrm{H}-4$ ).

Methyl 3-ethoxycarbonylmethoxy-1-ethoxycarbonylmethylindole-2-carboxylate $7 \mathrm{~b}$ :

Yield : $0.057 \mathrm{~g}(12 \%)$; oil. IR (film) $v=1740,1680(\mathrm{C}=\mathrm{O}) \mathrm{cm}^{-1} .{ }^{1} \mathrm{H}-\mathrm{NMR}\left(\mathrm{CDCl}_{3}\right) \delta=1.25\left(\mathrm{t}, 3 \mathrm{H}, \mathrm{J}=7.2, \mathrm{OCH}_{2} \mathrm{CH}_{3}\right), 1.31$ (t, $3 \mathrm{H}, \mathrm{J}=7.2, \mathrm{OCH}_{2} \mathrm{CH}_{3}$ ), $3.92\left(\mathrm{~s}, 3 \mathrm{H}, \mathrm{OCH}_{3}\right), 4.19$ (q, $\left.2 \mathrm{H}, \mathrm{J}=6.6, \mathrm{OCH}_{2}\right), 4.28\left(\mathrm{q}, 2 \mathrm{H}, \mathrm{J}=6.3, \mathrm{OCH}_{2}\right), 4.78\left(\mathrm{~s}, 2 \mathrm{H}, \mathrm{OCH}_{2}\right)$, $5.20\left(\mathrm{~s}, 2 \mathrm{H}, \mathrm{NCH}_{2}\right), 7.13-7.39\left(\mathrm{~m}, 3 \mathrm{H}, \mathrm{H}_{\text {arom }}\right), 7.85(\mathrm{~d}, 1 \mathrm{H}, \mathrm{J}=8.3, \mathrm{H}-7)$.

2-(2-Ethoxycarbonylethyl)-2-methoxycarbonyl-3-oxo-2,3-dihydroindole 9a :

Yield : $0.247 \mathrm{~g}(65 \%)$; oil. IR (film) $v=3380(\mathrm{br} \mathrm{NH}), 1750,1660(\mathrm{C}=0) \mathrm{cm}^{-1} .{ }^{1} \mathrm{H}-\mathrm{NMR}\left(\mathrm{CDCl}_{3}\right) \delta=1.18(\mathrm{t}, 3 \mathrm{H}, \mathrm{J}=6.9$, $\mathrm{OCH}_{2} \mathrm{CH}_{3}$ ), 2.26-2.56 (m, $4 \mathrm{H}, \mathrm{CH}_{2} \mathrm{CO}_{2} \mathrm{C}_{2} \mathrm{H}_{5}+\mathrm{CH}_{2} \mathrm{CH}_{2} \mathrm{CO}_{2} \mathrm{C}_{2} \mathrm{H}_{5}$ ), 3.72 (s, 3H, OCH$)_{3}$, 4.08 (quint., 2H, J=7.1, $\mathrm{OCH}_{2} \mathrm{CH}_{3}$ ), 5.40 (br s, $\left.1 \mathrm{H}, \mathrm{NH}\right), 6.89(\mathrm{t}, 1 \mathrm{H}, \mathrm{J}=7.4, \mathrm{H}-5), 6.97(\mathrm{~d}, 1 \mathrm{H}, \mathrm{J}=7.7, \mathrm{H}-7), 7.48(\mathrm{t}, 1 \mathrm{H}, \mathrm{J}=7.6, \mathrm{H}=6), 7.58$ (d, $1 \mathrm{H}$, $\mathrm{J}=7.5, \mathrm{H}-4)$.

2-(2-Cyanoethyl)-2-methoxycarbonyl-3-oxo-2,3-dihydroindole $9 \mathrm{~b}$ :

Yield : $0.214 \mathrm{~g}(67 \%)$; oil. IR (film) v=3380 (br NH), $2220(\mathrm{C} \equiv \mathrm{N}), 1730,1670(\mathrm{C}=\mathrm{O}) \mathrm{cm}^{-1} .{ }^{1} \mathrm{H}-\mathrm{NMR}\left(\mathrm{CDCl}_{3}\right) \delta=2.28-2.43$ (m, $4 \mathrm{H}, \mathrm{CH}_{2} \mathrm{CN}+\mathrm{CH}_{2} \mathrm{CH}_{2} \mathrm{CN}$ ), $3.72\left(\mathrm{~s}, 3 \mathrm{H}, \mathrm{OCH}_{3}\right.$ ), 5.34 (br s, $1 \mathrm{H}, \mathrm{NH}$ ), 6.87 (dd, 1H, J=7.8,7.3, H-5), 6.97 (d, 1H, J=7.9, $H-7), 7.46$ (dd, $1 H, J=7.3,7.9, H-6), 7.53$ (d, 1H, J=7.9, H-4).

2-(2-Methoxycarbonylethyl)-2-methoxycarbonyl-3-oxo-2,3-dihydroindole 10 :

Yield : $0.047 \mathrm{~g}(20 \%)$; oil. IR (film) v=3380 (br NH), 1750, $1680(\mathrm{C}=0) \mathrm{cm}^{-1} .{ }^{1} \mathrm{H}-\mathrm{NMR}\left(\mathrm{CDCl}_{3}\right) \delta=2.28-2.37(\mathrm{~m}, 2 \mathrm{H}$, $\mathrm{CH}_{2} \mathrm{CH}_{2} \mathrm{CO}_{2}$ ), 2.41-2.55 (m, 2H, CH $\mathrm{CO}_{2} \mathrm{CH}_{3}$ ), $3.64\left(\mathrm{~s}, 3 \mathrm{H}, \mathrm{OCH}_{3}\right), 3.77\left(\mathrm{~s}, 3 \mathrm{H}, \mathrm{OCH}_{3}\right), 6.90$ (dd, $\left.1 \mathrm{H}, \mathrm{J}=7.2,7.7, \mathrm{H}-5\right)$, $6.97(d, 1 H, J=8.6, H-7), 7.49$ (dd, $1 H, J=7.1,8.6, H-6), 7.59$ (d, $1 \mathrm{H}, J=7.9, H-4) . M S\left(N_{3}\right) m / z=278\left(M^{+}+1\right)$.

Methyl 3,9-dioxo-2,3,9,9a-tetrahydro-1H-pyrrolo[1,2-a]indole-9a-carboxylate 11 :

Yield : $0.038 \mathrm{~g}(25 \%)$; oil. IR (film) v=3380 (br NH), 1750, 1730, $1710(\mathrm{C}=0) \mathrm{cm}^{-1},{ }^{1} \mathrm{H}-\mathrm{NMR}\left(\mathrm{CDCl}_{3}\right) \delta=2.17-2.29(\mathrm{~m}$, $1 \mathrm{H}, \mathrm{NCCH}), 2.56-2.65(\mathrm{~m}, 1 \mathrm{H}, \mathrm{NCOCH}), 2.98-3.11(\mathrm{~m}, 2 \mathrm{H}, \mathrm{NCCH}+\mathrm{NCOCH}), 3.77\left(\mathrm{~s}, 3 \mathrm{H}, \mathrm{OCH}_{3}\right), 7.23-7.31(\mathrm{~m}, 1 \mathrm{H}, \mathrm{H}-4)$, 7.69-7.74 (m, 2H, H-5, H-6), $7.92(\mathrm{~d}, 1 \mathrm{H}, \mathrm{J}=7.9, \mathrm{H}-7) .{ }^{13} \mathrm{C}-\mathrm{NMR}\left(\mathrm{CDCl}_{3}\right) \delta=26.61\left(\mathrm{CCH}_{2}\right), 34.66\left(\mathrm{NCOCH}_{2}\right), 53.73$ $\left(\mathrm{OCH}_{3}\right), 76.51(\mathrm{NCCO}), 117.07\left(\mathrm{CH}_{\text {arom }}\right), 125.06\left(\mathrm{CH}_{\text {arom }}\right), 125.09\left(\mathrm{C}_{\text {arom }}\right), 125.28\left(\mathrm{CH}_{\text {arom }}\right), 137.47\left(\mathrm{CH}_{\text {arom }}\right), 151.13$ $\left(\mathrm{C}_{\text {arom }}\right), 167.54\left(\mathrm{CO}_{2} \mathrm{CH}_{3}\right), 173.07(\mathrm{NCO}), 194.08(\mathrm{CO}) . \mathrm{MS}\left(\mathrm{NH}_{3}\right) \mathrm{m} / \mathrm{z}=246\left(\mathrm{M}^{+}+1\right)$. 
Methyl 3-(3-cyanopropoxy)indole-2-carboxylate 13a :

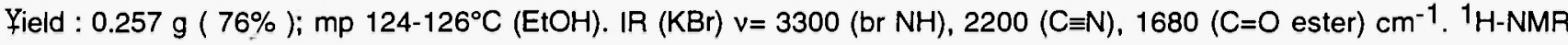
$\left(\mathrm{CDCl}_{3}\right) \delta=2.25$ (quint., $2 \mathrm{H}, \mathrm{J}=6.3, \mathrm{OCH}_{2} \mathrm{CH}_{2}$ ), $2.82\left(\mathrm{t}, 2 \mathrm{H}, \mathrm{J}=6.7, \mathrm{CH}_{2} \mathrm{CH}_{2} \mathrm{CN} \text { ), } 3.96 \text { (s, 3H, OCH}\right)_{3}, 4.23(\mathrm{t}, 2 \mathrm{H}, \mathrm{J}=5.5$, $\left.\mathrm{OCH}_{2}\right), 7.07-7.14\left(\mathrm{~m}, 2 \mathrm{H}, \mathrm{H}_{\text {arom }}\right), 7.36-7.38\left(\mathrm{~m}, 1 \mathrm{H}, \mathrm{H}_{\text {arom }}\right), 7.76(\mathrm{~d}, 1 \mathrm{H}, \mathrm{J}=8.2, \mathrm{H}-7), 8.36$ (br s, $\left.1 \mathrm{H}, \mathrm{NH}\right)$.

Methyl 3-(3-ethoxycarbonylpropoxy)indole-2-carboxylate 13b :

Yield : $0.280 \mathrm{~g}(70 \%) ; \mathrm{mp} 62-64^{\circ} \mathrm{C}(\mathrm{EtOH})$. IR $(\mathrm{KBr}) \mathrm{v}=3300(\mathrm{br} \mathrm{NH}), 1720\left(\mathrm{v}\right.$ br C=O ester) $\mathrm{cm}^{-1} .{ }^{1} \mathrm{H}-\mathrm{NMR}\left(\mathrm{CDCl}_{3}\right) \delta=$ 1.25 (t, 3H, J=7.4, OCH $\mathrm{CH}_{3}$ ), 2.14 (quint., $2 \mathrm{H}, \mathrm{J}=6.8, \mathrm{OCH}_{2} \mathrm{CH}_{2}$ ), 2.64 (t, $2 \mathrm{H}, \mathrm{J}=8.0, \mathrm{CH}_{2} \mathrm{CO}$ ), 3.97 (s, 3H, OCH$)_{3}, 4.14$ $\left(q, 2 \mathrm{H}, \mathrm{J}=6.9, \mathrm{OCH}_{2} \mathrm{CH}_{3}\right), 4.32\left(\mathrm{t}, 2 \mathrm{H}, \mathrm{J}=6.9, \mathrm{OCH}_{2}\right), 7.06-7.13\left(\mathrm{~m}, 4 \mathrm{H}, \mathrm{H}_{\text {arom }}\right)$.

Methyl [3-(3-cyanopropoxy)-1-(3-cyanopropyl)]indole-2-carboxylate 14a :

Yield : $0.213 \mathrm{~g}(50 \%)$; mp $86-88^{\circ} \mathrm{C}(\mathrm{EtOH})$. IR $(\mathrm{KBr}) \mathrm{v}=2220(\mathrm{C} \equiv \mathrm{N}), 1675\left(\mathrm{C}=\mathrm{O}\right.$ ester) $\mathrm{cm}^{-1}$. ${ }^{1} \mathrm{H}-\mathrm{NMR}\left(\mathrm{CDCl}_{3}\right) \delta=2.15$ (m, $4 \mathrm{H}, \mathrm{OCH}_{2} \mathrm{CH}_{2}+\mathrm{NCH}_{2} \mathrm{CH}_{2}$ ), $2.30\left(\mathrm{t}, 2 \mathrm{H}, \mathrm{J}=7.0, \mathrm{CH}_{2} \mathrm{CN}\right.$ ), $2.70\left(\mathrm{t}, 2 \mathrm{H}, \mathrm{J}=7.0, \mathrm{CH}_{2} \mathrm{CN}\right.$ ), $3.92\left(\mathrm{~s}, 3 \mathrm{H}, \mathrm{OCH}_{3}\right), 4.25(\mathrm{t}, 2 \mathrm{H}$, $\left.J=6.0, \mathrm{OCH}_{2}\right), 4.56\left(\mathrm{t}, 2 \mathrm{H}, \mathrm{J}=7.0, \mathrm{NCH}_{2}\right), 7.08-7.15\left(\mathrm{~m}, 2 \mathrm{H}, \mathrm{H}_{\text {arom }}\right), 7.36-7.37\left(\mathrm{~m}, 1 \mathrm{H}, \mathrm{H}_{\text {arom }}\right), 7.63(\mathrm{~d}, 1 \mathrm{H}, \mathrm{J}=8.0, \mathrm{H}-7)$.

Methyl [3-(3-ethoxycarbonylpropoxy)-1-(3-ethoxycarbonylpropyl)] indole-2-carboxylate 14b :

Yield : $0.329 \mathrm{~g}(60 \%)$; oil. IR (film) v=1725, $1690(\mathrm{C}=\mathrm{O}) \mathrm{cm}^{-1} .{ }^{1} \mathrm{H}-\mathrm{NMR}\left(\mathrm{CDCl}_{3}\right) \delta=1.20\left(\mathrm{t}, 3 \mathrm{H}, \mathrm{J}=7.0, \mathrm{OCH}_{2} \mathrm{CH}_{3}\right), 1.23$ (t, 3H, J=7.0, OCH $\mathrm{CH}_{3}$ ), 2.00-2.16 (m, $\left.4 \mathrm{H}, 2 \times \mathrm{CH}_{2} \mathrm{CH}_{2} \mathrm{CH}_{2}\right), 2.59$ (t, $\left.2 \mathrm{H}, \mathrm{J}=7.0, \mathrm{CH}_{2} \mathrm{CO}_{2}\right), 2.27$ (t, 2H, J=7.0, $\mathrm{CH}_{2} \mathrm{CO}_{2}$ ), $3.90\left(\mathrm{~s}, 3 \mathrm{H}, \mathrm{OCH}_{3}\right), 4.05-4.19\left(\mathrm{~m}, 4 \mathrm{H}, 2 \times \mathrm{OCH}_{2} \mathrm{CH}_{3}\right), 4.49\left(\mathrm{t}, 2 \mathrm{H}, \mathrm{J}=7.0, \mathrm{NCH}_{2}\right), 7.06$ (dd, $1 \mathrm{H}, \mathrm{J}=7.0,7.9, \mathrm{H}-5$ ), 7.29 (dd, $1 H, J=8.4,7.1, H-6), 7.36(d, 1 H, J=8.5, H-7), 7.66(d, 1 H, J=8.0, H-4)$.

Methyl 3-(3-cyanopropoxy)-1-methylindole-2-carboxylate 15a; Typical Procedure :

A solution of compound 13a $(5 \mathrm{~g}, 19.4 \mathrm{mmol})$ in dry THF (15 mL) was added slowly to a supension of sodium hydride $(0.698 \mathrm{~g}, 23.3 \mathrm{mmol})$ in dry THF $(5 \mathrm{~mL})$ at $0^{\circ} \mathrm{C}$. The mixture was stirred at this temperature for $1 \mathrm{~h}$, then iodomethane $(5.43$ $\mathrm{mL}, 87.3 \mathrm{mmol}$ ) was added. The reaction mixture was allowed to warm up to r.t. for $6 \mathrm{~h}$. The solvent was evaporated in vacuo, the mixture quenched with $\mathrm{H}_{2} \mathrm{O}$, neutralized with a solution of $10 \% \mathrm{HCl}$. The solution was extracted with $\mathrm{CH}_{2} \mathrm{Cl}_{2}$ $(3 \times 15 \mathrm{~mL})$, dried $\left(\mathrm{MgSO}_{4}\right)$ and the solvent evaporated in vacuo. Column chromatography on silica gel using $\mathrm{CH}_{2} \mathrm{Cl}_{2}$ as eluent afforded $15 \mathrm{a}$, yield : $4.08 \mathrm{~g}(77 \%) ; \mathrm{mp} 70-72^{\circ} \mathrm{C}(\mathrm{EtOH})$. IR $(\mathrm{KBr}) \mathrm{v}=2220(\mathrm{C} \equiv \mathrm{N}), 1680\left(\mathrm{C}=\mathrm{O}\right.$ ester) $\mathrm{cm}^{-1} .{ }^{1} \mathrm{H}-\mathrm{NMR}$ $\left(\mathrm{CDCl}_{3}\right) \delta=2.17$ (quint., $\left.2 \mathrm{H}, \mathrm{J}=6.2, \mathrm{OCH}_{2} \mathrm{CH}_{2}\right), 2.74\left(\mathrm{t}, 2 \mathrm{H}, \mathrm{J}=6.7, \mathrm{CH}_{2} \mathrm{CN}\right.$ ), 3.96 (s, 3H, NCH ), 3.97 (s, 3H, OCH $\mathrm{OCH}_{3}, 4.29$ $\left(\mathrm{t}, 2 \mathrm{H}, \mathrm{J}=5.5, \mathrm{OCH}_{2}\right), 7.10-7.16\left(\mathrm{~m}, 2 \mathrm{H}, \mathrm{H}_{\text {arom }}\right), 7.35-7.37\left(\mathrm{~m}, 1 \mathrm{H}, \mathrm{H}_{\text {arom }}\right), 7.67(\mathrm{~d}, 1 \mathrm{H}, \mathrm{J}=8.5, \mathrm{H}-7)$.

Methyl 3-(3-ethoxycarbonylpropoxy)-1-methylindole-2-carboxylate 15b :

$Y$ ield : $4.13 \mathrm{~g}(79 \%)$; oil. IR (film) $v=1720,1680$ (C=O ester) $\mathrm{cm}^{-1}$. ${ }^{1} \mathrm{H}-\mathrm{NMR}\left(\mathrm{CDCl}_{3}\right) \delta=1.27\left(\mathrm{t}, 3 \mathrm{H}, \mathrm{J}=7.9, \mathrm{OCH}_{2} \mathrm{CH}_{3}\right)$, 2.14 (quint., $\left.2 \mathrm{H}, \mathrm{J}=7.1, \mathrm{OCH}_{2} \mathrm{CH}_{2}\right), 2.63\left(\mathrm{t}, 2 \mathrm{H}, \mathrm{J}=7.9, \mathrm{CH}_{2} \mathrm{CO}\right), 3.94\left(\mathrm{~s}, 3 \mathrm{H}, \mathrm{NCH}_{3}\right), 3.97\left(\mathrm{~s}, 3 \mathrm{H}, \mathrm{OCH}_{3}\right), 4.12-4.23(\mathrm{~m}$, $\left.4 \mathrm{H}, \mathrm{OCH}_{2}+\mathrm{OCH}_{2} \mathrm{CH}_{3}\right), 7.06-7.12\left(\mathrm{~m}, 2 \mathrm{H}, \mathrm{H}_{\text {arom }}\right), 7.30-7.36\left(\mathrm{~m}, 1 \mathrm{H}, \mathrm{H}_{\text {arom }}\right), 7.70(\mathrm{~d}, 1 \mathrm{H}, \mathrm{J}=8.1, \mathrm{H}-7)$.

4-Cyano-6-methyl-5-oxo-2,3,4,5-tetrahydrooxepino[3,2-b]indole 16a; Typical Procedure :

To a stirred solution of diisopropylamine $(0.77 \mathrm{~mL}, 5.5 \mathrm{mmol})$ in dry THF $(10 \mathrm{~mL})$ was added dropwise butyllithium (1.6 M in hexane, $3.44 \mathrm{~mL}, 5.5 \mathrm{mmol}$ ) at $-78^{\circ} \mathrm{C}$ under nitrogen atmosphere and the mixture was stirred for $30 \mathrm{~min}$. A solution of 
the compound $15 \mathrm{a}(1.5 \mathrm{~g}, 5.5 \mathrm{mmol})$ in dry THF $(10 \mathrm{~mL})$ was added dropwise and the mixture was stirred at this temperature for $45 \mathrm{~min}$. The solution was allowed to warm up to r.t. for $3 \mathrm{~h}$. The solvent was evaporated in vacuo, the mixture quenched with $\mathrm{H}_{2} \mathrm{O}$, neutralized with a solution of $10 \% \mathrm{HCl}$. The solution was extracted with $\mathrm{CH}_{2} \mathrm{Cl}_{2}(2 \times 15 \mathrm{~mL})$, dried $\left(\mathrm{MgSO}_{4}\right)$ and the solvent evaporated in vacuo. Column chromatography on silica gel eluting with $\mathrm{CH}_{2} \mathrm{Cl}_{2}$ afforded 16a, yield : $1.03 \mathrm{~g}(78 \%)$; mp 120-122 $\mathrm{C}(\mathrm{EtOH})$. IR $(\mathrm{KBr}) \mathrm{v}=2200(\mathrm{C} \equiv \mathrm{N}), 1650(\mathrm{C}=\mathrm{O}) \mathrm{cm}^{-1} .{ }^{1} \mathrm{H}-\mathrm{NMR}\left(\mathrm{CDCl}_{3}\right) \delta=2.44-$ $2.71\left(\mathrm{~m}, 2 \mathrm{H}, \mathrm{OCH}_{2} \mathrm{CH}_{2}\right), 3.91\left(\mathrm{~s}, 3 \mathrm{H}, \mathrm{NCH}_{3}\right), 4.88(\mathrm{q}, 1 \mathrm{H}, \mathrm{J}=4.5, \mathrm{CHCN}), 4.40-4.57\left(\mathrm{~m}, 2 \mathrm{H}, \mathrm{OCH}_{2}\right), 7.10(\mathrm{t}, 1 \mathrm{H}, \mathrm{J}=7.6, \mathrm{H}-$ 5), 7.25-7.30 (m, $\left.2 \mathrm{H}, \mathrm{H}_{\text {arom }}\right), 7.40-7.46\left(\mathrm{~m}, 1 \mathrm{H}_{3} \mathrm{H}_{\text {arom }}\right), 7.70(\mathrm{~d}, 1 \mathrm{H}, \mathrm{J}=8.2, \mathrm{H}-7)$. MS $\left(\mathrm{NH}_{3}\right) \mathrm{m} / z=241\left(\mathrm{M}^{+}+1\right)$.

\section{4-Ethoxycarbonyl-12-methyl-5-oxo-2,3,4,5-tetrahydrooxepino[3,2-b]indole 16b :}

Yield : $1.00 \mathrm{~g}(75 \%)$; oil. IR (film) v= $1730\left(\mathrm{C}=\mathrm{O}\right.$ ester), $1640(\mathrm{C}=\mathrm{O}) \mathrm{cm}^{-1} .{ }^{1} \mathrm{H}-\mathrm{NMR}\left(\mathrm{CDCl}_{3}\right) \delta=1.26(\mathrm{t}, 3 \mathrm{H}, \mathrm{J}=6.2$, $\mathrm{OCH}_{2} \mathrm{CH}_{3}$ ), 2.36-2.63 (m, $\left.2 \mathrm{H}, \mathrm{OCH}_{2} \mathrm{CH}_{2}\right), 2.87\left(\mathrm{t}, 1 \mathrm{H}, \mathrm{J}=3.8, \mathrm{CHCO}_{2}\right), 3.90\left(\mathrm{~s}, 3 \mathrm{H}, \mathrm{NCH}_{3}\right), 4.24$ (quint., 2H, J=6.9, $\left.\mathrm{OCH}_{2}\right), 4.37-4.51\left(\mathrm{~m}, 2 \mathrm{H}, \mathrm{OCH}_{2}\right), 7.06(\mathrm{t}, 1 \mathrm{H}, \mathrm{J}=7.1, \mathrm{H}-5), 7.24(\mathrm{~d}, 1 \mathrm{H}, \mathrm{J}=8.5, \mathrm{H}-7), 7.38(\mathrm{t}, 1 \mathrm{H}, \mathrm{J}=8.5, \mathrm{H}-6), 7.67(\mathrm{~d}, 1 \mathrm{H}$, $J=7.8, \mathrm{H}-4) . \mathrm{MS}\left(\mathrm{NH}_{3}\right) \mathrm{m} / \mathrm{z}=288\left(\mathrm{M}^{+}+1\right)$.

4-Amino-12-methyl-5,6-dihydroindolo[3,2-b]oxepino[5,4-o]pyrimidine 17 :

A mixture of $16 \mathrm{a}(0.200 \mathrm{~g}, 0.83 \mathrm{mmol})$ and formamide $(10 \mathrm{~mL})$ was heated at $140-150^{\circ} \mathrm{C}$ with stirring under $\mathrm{NH}_{3}$ stream for $36 \mathrm{~h}$ until the substrate $16 \mathrm{a}$ has disappeared. The mixture was cooled and quenched with $\mathrm{H}_{2} \mathrm{O}$. It was extracted with $\mathrm{CH}_{2} \mathrm{Cl}_{2}(3 \times 10 \mathrm{~mL})$, dried $\left(\mathrm{MgSO}_{4}\right)$ and the solvent was evaporated in vacuo. Column chromatography on silica gel $\left(\mathrm{CH}_{2} \mathrm{Cl}_{2} / \mathrm{MeOH}, 99: 1\right)$ gave 17, yield : $0.121 \mathrm{~g}(55 \%)$; mp 166-168 ${ }^{\circ} \mathrm{C}(\mathrm{EtOH}) . \mathrm{IR}(\mathrm{KBr}) \mathrm{v}=3400\left(\mathrm{br} \mathrm{NH}_{2}\right) \mathrm{cm}^{-1} .{ }^{1} \mathrm{H}-\mathrm{NMR}$ $\left(\mathrm{CDCl}_{3}\right) \delta=2.94\left(\mathrm{t}, 2 \mathrm{H}, \mathrm{J}=5.9, \mathrm{OCH}_{2} \mathrm{CH}_{2}\right), 4.03\left(\mathrm{~s}, 3 \mathrm{H}, \mathrm{NCH}_{3}\right), 4.54\left(\mathrm{t}, 2 \mathrm{H}, \mathrm{J}=5.9, \mathrm{OCH}_{2}\right), 5.00\left(\mathrm{br} \mathrm{s}, 2 \mathrm{H}, \mathrm{NH}_{2}\right), 7.05-7.10$ $\left(m, 2 H, H_{\text {arom }}\right), 7.32(d, 1 H, J=8.3, H-7), 7.65(d, 1 H, J=7.9, H-4), 8.54(\mathrm{~s}, 1 \mathrm{H}, \mathrm{N}=\mathrm{CH}) . M S\left(\mathrm{NH}_{3}\right) \mathrm{m} / z=267\left(\mathrm{M}^{+}+1\right)$.

Diethyl N-(5,6-dihydro-12-methylindolo[3,2-b]oxepino[5,4-d]pyrimidin-4-yl)aminomethylene malonate 18 :

A mixture of compound $17(0.050 \mathrm{~g}, 0.19 \mathrm{mmol})$ and diethyl ethoxymethylenemalonate $(0.1 \mathrm{~mL}, 0.38 \mathrm{mmol})$ in xylene (5 $\mathrm{mL}$ ) was refluxed for $18 \mathrm{~h}$. After evaporation of the reaction mixture, the residue was chromatographed on silica gel using petroleum ether/ethyle acetate $9: 1$ as eluent to give 18 , yield : $0.060 \mathrm{~g}(73 \%)$; mp $180-182^{\circ} \mathrm{C}(\mathrm{EtOH}) . \mathrm{IR}(\mathrm{KBr}) \mathrm{v}=3180$ (br $\mathrm{NH}), 1720(\mathrm{C}=\mathrm{O}), 1650(\mathrm{C}=\mathrm{C}) \mathrm{cm}^{-1} .{ }^{1} \mathrm{H}-\mathrm{NMR}\left(\mathrm{CDCl}_{3}\right) \delta=1.30\left(\mathrm{t}, 3 \mathrm{H}, \mathrm{J}=7.3, \mathrm{OCH}_{2} \mathrm{CH}_{3}\right), 1.35$ (t, 3H, J=7.3, $\mathrm{OCH}_{2} \mathrm{CH}_{3}$ ), 3.04-3.11 (m, $2 \mathrm{H}, \mathrm{OCH}_{2} \mathrm{CH}_{2}$ ), $3.97\left(\mathrm{~s}, 3 \mathrm{H}, \mathrm{NCH}_{3}\right), 4.24$ (q, 2H, J=7.3, OCH$)_{2}, 4.30$ (q, 2H, J=7.3, OCH $)$, 4.48-4.55 (m, 2H, OCH$), 7.00-7.35\left(\mathrm{~m}, 3 \mathrm{H}, \mathrm{H}_{\text {arom }}\right), 7.62(\mathrm{~d}, 1 \mathrm{H}, \mathrm{J}=8.1, \mathrm{H}-7), 8.76(\mathrm{~s}, 1 \mathrm{H}, \mathrm{N}=\mathrm{CH}), 9.24(\mathrm{~d}, 1 \mathrm{H}, \mathrm{J}=11.7$, $=\mathrm{CH}), 11.62(\mathrm{brd}, 1 \mathrm{H}, \mathrm{NH}) . \mathrm{MS}\left(\mathrm{NH}_{3}\right) \mathrm{m} / \mathrm{z}=437\left(\mathrm{M}^{+}+1\right)$.

Ethyl 1-oxo-5,6-dihydro-12-methylindolo[3,2-b]oxepino[4,5-e]pyrimido[1,2-c]pyrimidin-2-yl carboxylate 19 : Compound $18(0.040 \mathrm{~g}, 0.09 \mathrm{mmol})$ in diphenyl ether $(5 \mathrm{~mL})$ was heated at $250-260^{\circ} \mathrm{C}$ with a metallic bath for $90 \mathrm{mn}$. After cooling of the reaction mixture, petroleum ether was added to the mixture. The precipitated solid was collected by filtration, washed with petroleum ether and dried in vacuo to afford 19 , yield : $0.031 \mathrm{~g}(86 \%) ; \mathrm{mp} 220-222^{\circ} \mathrm{C}(\mathrm{EtOH}) . \mathrm{IR}(\mathrm{KBr}) \mathrm{v}=$ $1710\left(\mathrm{C}=\mathrm{O}\right.$ ester), $1680(\mathrm{C}=\mathrm{O}), 1610(\mathrm{C}=\mathrm{C}) \mathrm{cm}^{-1} .{ }^{1} \mathrm{H}-\mathrm{NMR}\left(\mathrm{CDCl}_{3}\right) \delta=1.37\left(\mathrm{t}, 3 \mathrm{H}, \mathrm{J}=7.5, \mathrm{OCH}_{2} \mathrm{CH}_{3}\right), 3.58-3.67(\mathrm{~m}, 2 \mathrm{H}$, $\left.\mathrm{OCH}_{2} \mathrm{CH}_{2}\right), 3.98\left(\mathrm{~s}, 3 \mathrm{H}, \mathrm{NCH}_{3}\right), 4.37$ (q, $\left.2 \mathrm{H}, \mathrm{J}=7.5, \mathrm{OCH}_{2}\right), 4.47-4.58\left(\mathrm{~m}, 2 \mathrm{H}, \mathrm{OCH}_{2}\right), 6.99-7.40\left(\mathrm{~m}, 3 \mathrm{H}, \mathrm{H}_{\text {arom }}\right), 7.62(\mathrm{~d}$, $1 \mathrm{H}, \mathrm{J}=8.1, \mathrm{H}-7), 8.95(\mathrm{~s}, 1 \mathrm{H},=\mathrm{CH}), 9.78(\mathrm{~s}, 1 \mathrm{H}, \mathrm{N}=\mathrm{CH}) . \mathrm{MS}\left(\mathrm{NH}_{3}\right) \mathrm{m} / \mathrm{z}=391\left(\mathrm{M}^{+}+1\right)$. 
4-Acetamido-5,6-dihydro-12-methylindolo[3,2-b]oxepino[5,4-d]pyrimidine 20 :

The pyrimidine $17(0.040 \mathrm{~g}, 0.15 \mathrm{mmol})$ was dissolved in $\mathrm{CH}_{2} \mathrm{Cl}_{2}(5 \mathrm{~mL})$ and cooled to $0^{\circ} \mathrm{C}$ with an ice-bath, under argon atmosphere. Acetyl chloride $(0.01 \mathrm{~mL}, 0.16 \mathrm{mmol})$-and triethylamine $(0.02 \mathrm{~mL}, 0.16 \mathrm{mmol})$ were added slowly. The mixture was stirred at r.t. for $18 \mathrm{~h}$, hydrolyzed and extracted with $\mathrm{CH}_{2} \mathrm{Cl}_{2}(2 \times 5 \mathrm{~mL})$. The organic extracts were washed with $\mathrm{H}_{2} \mathrm{O}$, dried $\left(\mathrm{MgSO}_{4}\right)$ and evaporated under reduced pressure. The residue was chromatographed over silica gel using $\mathrm{CH}_{2} \mathrm{Cl}_{2}$ to give 20, yield : $0.036 \mathrm{~g}(78 \%)$; mp 188-190 ${ }^{\circ} \mathrm{C}(\mathrm{EtOH})$. IR (KBr) v=3400 (br NH), $1710(\mathrm{C}=0) \mathrm{cm}^{-1} .{ }^{1} \mathrm{H}-\mathrm{NMR}\left(\mathrm{CDCl}_{3}\right) \delta$ $=2.31\left(\mathrm{~s}, 3 \mathrm{H}, \mathrm{COCH}_{3}\right), 2.91\left(\mathrm{t}, 2 \mathrm{H}, \mathrm{J}=4.4, \mathrm{OCH}_{2} \mathrm{CH}_{2}\right), 4.02\left(\mathrm{~s}, 3 \mathrm{H}, \mathrm{NCH}_{3}\right), 4.40\left(\mathrm{t}, 2 \mathrm{H}, \mathrm{J}=4.4, \mathrm{OCH}_{2}\right), 6.99-7.38(\mathrm{~m}, 3 \mathrm{H}$, $\left.\mathrm{H}_{\text {arom }}\right), 7.62(\mathrm{~d}, 1 \mathrm{H}, \mathrm{J}=8.1, \mathrm{H}-7), 8.99(\mathrm{~s}, 1 \mathrm{H}, \mathrm{N}=\mathrm{CH}) . \mathrm{MS}\left(\mathrm{NH}_{3}\right) \mathrm{m} / \mathrm{z}=309\left(\mathrm{M}^{+}+1\right)$.

Ethyl 3-(3-cyanopropoxy)-1-methylindole-2-carboxylate 23 :

$Y$ ield : $0.248 \mathrm{~g}(76 \%)$; oil. IR (film) v= $2200(\mathrm{C} \equiv \mathrm{N}), 1680\left(\mathrm{C}=\mathrm{O}\right.$ ester) $\mathrm{cm}^{-1} .{ }^{1} \mathrm{H}-\mathrm{NMR}\left(\mathrm{CDCl}_{3}\right) \delta=1.43(\mathrm{t}, 3 \mathrm{H}, \mathrm{J}=7.1$, $\mathrm{OCH}_{2} \mathrm{CH}_{3}$ ), 2.15 (quint., $2 \mathrm{H}, \mathrm{J}=6.3, \mathrm{OCH}_{2} \mathrm{CH}_{2}$ ), 2.74 (t, $2 \mathrm{H}, \mathrm{J}=6.7, \mathrm{CH}_{2} \mathrm{CN}$ ), 3.96 (s, 3H, $\mathrm{NCH}_{3}$ ), 4.29 (t, 2H, J=5.5, $\left.\mathrm{OCH}_{2}\right), 4.43\left(\mathrm{q}, 2 \mathrm{H}, \mathrm{J}=7.1, \mathrm{OCH}_{2}\right), 7.10-7.16\left(\mathrm{~m}, 2 \mathrm{H}, \mathrm{H}_{\text {arom }}\right), 7.35-7.37\left(\mathrm{~m}, 1 \mathrm{H}, \mathrm{H}_{\text {arom }}\right), 7.67(\mathrm{~d}, 1 \mathrm{H}, \mathrm{J}=8.5, \mathrm{H}-7)$.

\section{References :}

(1) A. Etienne, Bull. Soc. Chim. Fr. 651 (1948)

(2) P.C. Unangst, R.E. Brown, D.J. Herzig, J. Med. Chem. 233, 1251 (1980)

(3) P.C. Unangst, R.E. Brown, J. Heterocycl. Chem. 21, 283 (1984)

(4) P.C. Unangst, R.E. Brown, A. Fabian, F. Fontsere, J. Heterocycl. Chem. 16, 661 (1979)

(5) R.E. Brown, US Patent 4013641 (March 13, 1977); Chem. Abstr. 8Z, 39553d (1977)

(6) T. Nagamatsu, S. Tsurubayashi, K. Sasaki, T. Hirota, Synthesis 303 (1991)

(7) T. Nagamatsu, K. Kinoshita, K. Sasaki, T. Nakayama, T. Hirota, J. Heterocycl. Chem. 28 , 513 (1991)

(8) B.M. Trost, I. Fleming, Comprehensive Organic Synthesis, Pergamon Press, Vol 3, pp 54 (1991)

(9) J.Y. Merour, F. Cossais, Synth. Comm. 23, 1813 (1993)

(10) H. Plieninger, H. Sirowej, D. Rau, Chem. Ber. 104, 1863 (1971)

(11) E. Houghton, J.E. Saxton, J. Chem. Soc. (C) 595 (1969)

(12) P.C. Unangst, D.T. Connor, S.R. Stabler, R.J. Weikert, J. Heterocycl. Chem. 24, 811 (1987)

(13) T. Nagamatsu, Y. Hantani, K. Sasaki, H. Ohtomo, T. Nakayama, T. Hirota, J. Heterocycl. Chem. 30, 233 (1993)

(14) K. Sasaki, Y. Arimoto, H. Ohtomo, T. Nakayama, T. Hirota, J. Heterocycl. Chem. 30, 989 (1993)

(15) P.C. Unangst, M.E. Carethers, J. Heterocycl. Chem. 21, 709 (1984)

(16) J.Y. Merour, L. Chichereau, B. Malapel, unpublished results

(17) K. Sasaki, Y. Tashima, T. Nakayama, T. Hirota, J. Heterocycl. Chem. 28, 269 (1991)

(18) These experiments have been performed by $P$. Chaimbault (undergraduate summer student)

(19) T. Nagamatsu, Y. Hantani, M. Takaishi, K. Sasaki, T. Hirota, Synthesis 942 (1991)

Received September 21, 1994 
